\section{Kein Erwerb der Kompetenz Magnetresonanz- tomographie durch Fachärzte für Orthopädie und Unfallchirurgie im Rahmen der Weiterbildung (Teil 2)}

In dem ersten Teil dieses Beitrages wurde die Frage der privatärztlichen Erbringung und Abrechenbarkeit von MRT-Leistungen durch Fachärzte/Fachärztinnen für Orthopädie nach den bisherigen Weiterbildungsordnungen der Landesärztekammern dargestellt.

Der hier folgende zweite Teil beschäftigt sich mit der Frage, ob sich an dieser Beurteilung etwas durch die neue Struktur der Weiterbildung in der (Muster-)Weiterbildungsordnung der Bundesärztekammer von 2018 (MWBO 2018) geändert hat.

\section{d. Fachgebietskonformität von MRT-Leistungen für Fachärzte/ Fachärztinnen für Orthopädie und Unfallchirurgie nach der MWBO 2018}

Die neue MWBO 2018, wurde am 05./ 16.11.2018 beschlossen (https://www. bundesaerztekammer.de/aerzte/ausweiter-fortbildung/weiterbildung/musterweiterbildungsordnung/).

In der MWBO 2018 wird nun, bezogen auf die Weiterbildungsinhalte der Facharzt-Kompetenz zwischen „Kognitive und Methodenkompetenz“ (Kenntnisse) und „Handlungskompetenz“ (Erfahrungen und Fertigkeiten) differenziert. Für den Facharzt/Fachärztin für Orthopädie und Unfallchirurgie werden im Bereich der Diagnostischen Verfahren in der Methodenkompetenz allerdings keine Verfahren genannt ( $>$ Tab. 1).

In der Handlungskompetenz werden u. a. folgende Weiterbildungsinhalte für Fachärzte/Fachärztinnen für Orthopädie und Unfallchirurgie aufgeführt ( $\triangleright$ Tab. 2).

Aus der Beschreibung der diagnostischen Verfahren für die Facharzt-Kompetenz Orthopädie und Unfallchirurgie in der MWBO 2018 wird deutlich, dass bestimmte definierte diagnostische Verfahren, dann zum Fachgebiet des Facharztes/Fachärztin für Orthopädie und Unfallchirurgie gehören, wenn auch die „Durchführung“ dieser diagnostischen Verfahren Gegenstand der Handlungskompetenz ist, diese damit als erlernt gelten können und hierfür auch entsprechende Richtzahlen vorgegeben sind. Hierzu gehören z. B. die Sonografie, die konventionelle Röntgendiagnostik und die Osteodensitometrie. Eine weitere Handlungskompetenz besteht im Bereich der diagnostischen Verfahren für das Fachgebiet Orthopädie und Unfallchirurgie nur im Bereich der „Indikationsstellung und Befundinterpretation weiterer bildgebender Verfahren“.

Folglich gilt auch nach der aktuellen MWBO 2018 der Bundesärztekammer, dass der Facharzt für Orthopädie und Unfallchirurgie gerade keine Berechtigung zur Durchführung anderer als der zuvor genannten diagnostischen Verfahren besitzt, sondern lediglich die Indikationsstellung und die Befundinterpretation für MRT-Leistungen

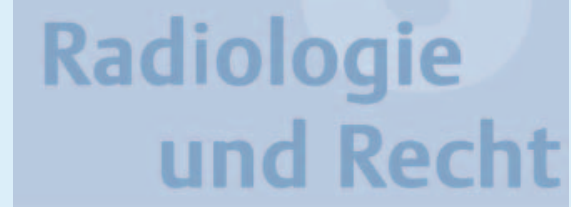

gebietskonform durchführen kann. Damit wird auch durch die MWBO 2018 bestätigt, dass der Facharzt für Orthopädie und Unfallchirurgie keine Handlungskompetenz im Sinne von Erfahrungen und Fertigkeiten zur Durchführung von MRT-Untersuchungen im Gebiet besitzt.

Wäre demgegenüber die Auffassung der Bayerischen Landesärztekammer (BLÄK) und der Landgerichte Regensburg und Landshut zutreffend, wonach sich bereits aus den allgemeinen Begriffen der „Vorbeugung, Erkennung, konservative und operative Behandlung, Nachsorge und Rehabilitation " in der Gebietsbezeichnung der Chirurgie die Gebietszugehörigkeit sämtli-

- Tab. 1 Facharzt/Fachärztin für Orthopädie und Unfallchirurgie

\begin{tabular}{l|l|l|}
$\begin{array}{l}\text { Kognitive und Methodenkompetenz } \\
\text { Kenntnisse }\end{array}$ & $\begin{array}{l}\text { Handlungskompetenz } \\
\text { Erfahrungen und Fertigkeiten }\end{array}$ & Richtzahl \\
\hline
\end{tabular}

Diagnostische Verfahren

Richtungsweisende Sonografie der Säuglingshüfte

Durchführung und Befunderstellung von Ultraschalluntersuchungen, davon

- Notfallsonografien (eFast) 50

- Am Bewegungsapparat einschließlich 50 Arthrosonografien

Indikation, Durchführung und

Befunderstellung von konventioneller Röntgendiagnostik

- Notfalldiagnostik: Röntgendiagnostik ohne CT im Rahmen der Erstversorgung bei Erwachsenen und Kindern

(Muster-Weiterbildungsordnung 2018, Weiterbildungsinhalte der Facharzt-Kompetenz Orthopädie und Unfallchirurgie) 
- Tab. 2 Facharzt/Fachärztin für Orthopädie und Unfallchirurgie

\begin{tabular}{|l|l|l|}
$\begin{array}{l}\text { Kognitive und Methodenkompetenz } \\
\text { Kenntnisse }\end{array}$ & $\begin{array}{l}\text { Handlungskompetenz } \\
\text { Erfahrungen und Fertigkeiten }\end{array}$ & Richtzahl \\
\hline & - Am Skelett \\
\hline & $\begin{array}{l}\text { - Intraoperative radiologische } \\
\text { Befundkontrolle }\end{array}$ & \\
\hline & Indkatonsstelung und Befunditerpe- \\
\hline
\end{tabular}

Indikationsstellung und Befundinterpretation weiterer bildgebender Verfahren Indikation, Durchführung und Befunderstellung der Osteodensitometrie

Indikationsstellung und Befundinterpretation neurophysiologischer Diagnostik

(Muster-Weiterbildungsordnung 2018, Weiterbildungsinhalte der Facharzt-Kompetenz Orthopädie und Unfallchirurgie)

- Tab. 3 Facharzt/Fachärztin für Radiologie

\begin{tabular}{l|l|l|}
$\begin{array}{l}\text { Kognitive und Methodenkompetenz } \\
\text { Kenntnisse }\end{array}$ & $\begin{array}{l}\text { Handlungskompetenz } \\
\text { Erfahrungen und Fertigkeiten }\end{array}$ & Richtzahl \\
\hline Magnetresonanztomographie & & \\
\hline $\begin{array}{l}\text { Prinzipien von Magnetfeldstärke, } \\
\text { Gradientenstärke, Hochfrequenz, Orts- } \\
\text { und Zeitauflösung }\end{array}$ & \\
\hline $\begin{array}{l}\text { Gerätebezogene Sicherheitsvorschrif- } \\
\text { ten in Bezug auf Personal und Patienten }\end{array}$ & \\
\hline $\begin{array}{l}\text { Typische Artefakte in der MRT und ihre } \\
\text { Ursachen }\end{array}$ & \\
\hline
\end{tabular}

Grundlagen der Gefäßdarstellung und funktioneller MRT-Techniken

Indikation für PET/MRT im Kontext multimodaler Bildgebung

Indikation, Durchführung und Befunder3.000 stellung von MRT-Untersuchungen aller Körperregionen, z. B. ZNS, Nerven, muskuloskelettales System, Weichteile, Thorax, Herz, Abdomen, Becken, Gefäße, fetale MRT, MRT-Interventionen

Erstellung und Anwendung von MRTUntersuchungsprotokollen für alle Körperregionen und alle MR-Verfahren einschließlich geeigneter Kontrastmittel

(Muster-Weiterbildungsordnung 2018, Weiterbildungsinhalte der Facharzt-Kompetenz Radiologie)

cher Methoden und Verfahren ergeben würde, müsste der Facharzt für Orthopädie und Unfallchirurgie z. B. auch berechtigt sein, die „Sonografie der Thoraxorgane und der thorakalen Gefäße einschließlich Dopplerund Duplexuntersuchungen“, die mit einer
Richtzahl von 200 versehen ist, durchzuführen, obwohl die Handlungskompetenz für dieses diagnostische Verfahren ausdrücklich nur in den Weiterbildungsinhalten des Facharztes für Herzchirurgie beschrieben wird.
Auch wenn nach §2 Abs. 2 S. 4 MWBO bestimmt wird, dass die in der Facharztkompetenz vorgeschriebenen Weiterbildungsinhalte nicht die Ausübung der fachärztlichen Tätigkeit im Gebiet beschränken, ist doch anerkannt, dass ein Arzt, der eine Facharztbezeichnung führt, nicht alle Leistungen erbringen darf, die dieses Fachgebiet qua Definition umfasst. Deshalb darf z. B. ein Facharzt für Allgemeinchirurgie nicht herzchirurgisch tätig sein, sondern nur dann, wenn er die entsprechende Kompetenz für die betreffenden Leistungen erworben hat (vgl. Hübner, Ärztliches Berufsrecht, 2018, 2. Kapitel, VII, 4.1, RdNr. W 71).

Wenn es danach aber bereits nicht hinzunehmen ist, dass ein Arzt, der eine Facharztbezeichnung führt, alle Leistungen erbringt, die dieses Fachgebiet qua Definition und in den Schwerpunkten umfasst, muss erst Recht im Verhältnis der Fachgebiete untereinander der Grundsatz gelten, dass ein Facharzt Leistungen nur dann erbringen darf, wenn er die entsprechende Kompetenz erworben hat.

Die kognitive Methodenkompetenz und die Handlungskompetenz zur Durchführung von MRT-Untersuchungen besteht nach der MWBO 2018 weiterhin nur für den Facharzt/Fachärztin für Radiologie, wobei die Inhalte weiter präzisiert worden sind ( Tab. 3)

Im Bereich der Handlungskompetenz wird für das Gebiet Radiologie nun hervorgehoben, dass der Facharzt/Fachärztin für Radiologie die Berechtigung zur „Indikation, Durchführung und Befunderstellung von MRT-Untersuchungen aller Körperregionen, z. B. ZNS, Nerven, muskuloskelettales System, Weichteile, Thorax, Herz, Abdomen, Becken, Gefäße, fetale MRT, MRT-Interventionen “ besitzt. Damit sind gerade auch die von dem Facharzt/Fachärztin für Orthopädie und Unfallchirurgie im Rahmen der Zusatzweiterbildung erlernbaren MRTUntersuchungen des sog. muskuloskelettales System gebietsbezogen ausschließlich dem Fachgebiet der Radiologie zugeordnet. 


\section{Die Bedeutung der Zusatz-} Weiterbildung Magnetresonanztomographie - fachgebunden - für die Beurteilung
der Fachgebietskonformität

Diese Einschätzung wird auch durch die Vorgaben der Zusatz-Weiterbildung Magnetresonanztomographie - fachgebunden - gestützt. Nach deren Definition umfasst die ZusatzWeiterbildung in Ergänzung zu einer Facharztkompetenz die Durchführung und Befundung gebietsbezogener Bildgebungsverfahren mittels MRT. Ziel ist die Erlangung der fachlichen Kompetenz in fachgebundener MRT.

Eine Zusatz-Weiterbildung hat nach $\S 2$ Abs. 4 der Weiterbildungsordnung der BLÄK folgende Ziele:

„(4) Zusatz-Weiterbildung im Sinn dieser Weiterbildungsordnung ist insbesondere ein Bereich im Sinn des Abschnittes IV des Heilberufe-Kammergesetzes und Spezialisierung in Weiterbildungsinhalten (zusätzliche Kenntnisse und Fähigkeiten), die nach Maßgabe des Abschnittes $C$ zusätzlich zu einer oder ohne eine Facharzt- und Schwerpunktweiterbildung erworben werden kann. Sind in Abschnitt C Weiterbildungszeiten verlangt, dürfen diese nicht gleichzeitig mit der Facharzt- oder Schwerpunktweiterbildung abgeleistet werden, sofern in Abschnitt C nichts anderes festgelegt ist. Die Gebietsgrenzen werden durch eine ZusatzWeiterbildung nicht erweitert. [...]“

In der Zusatz-Weiterbildung 20. Magnetresonanztomographie - fachgebunden - der BLÄK wird das Weiterbildungsziel daher wie folgt definiert:

„Ziel der Zusatz-Weiterbildung ist die Erlangung der fachlichen Kompetenz in fachgebundener Magnetresonanztomographie nach Ableistung der vorgeschriebenen Weiterbildungszeit und Weiterbildungsinhalte“

Neben der erforderlichen Weiterbildungszeit von 24 Monaten, die bei einem Weiterbilder für Radiologie abgeleistet werden muss, wird in den Weiterbildungsrichtlinien der BLÄK für die Facharztkompetenz Orthopädie und Unfallchirurgie eine Richtzahl von 1.000 gebietsbezogenen Magnetresonanztomographien gefordert.
Gerade die für die Zusatz-Weiterbildung „Magnetresonanztomographie - fachgebunden -“ einerseits geforderte Richtzahl von 1.000 Untersuchungen sowie die mangelnde Erwähnung der Magnetresonanztomographie in der Gebietsbezeichnung und die fehlenden Richtzahlen für das Fachgebiet der Chirurgie und des Facharztes/Fachärztin für Orthopädie und Unfallchirurgie andererseits machen deutlich, dass die Methode der MRT nicht zu diesem Fachgebiet gehört. Sofern eine Methode Gegenstand der Facharztweiterbildung ist, werden regelmäßig nach den Weiterbildungsrichtlinien Weiterbildungszeiten und Richtzahlen zum Erwerb der erforderlichen Kenntnisse gefordert. Diese Systematik der WBO wird gerade für die Facharztkompetenz Orthopädie und Unfallchirurgie dadurch besonders verdeutlicht, dass für die ZusatzWeiterbildung Magnetresonanztomographie - fachgebunden - die Durchführung und Befundung von 1.000 MRT-Untersuchungen gefordert wird, während in der Gebietsbezeichnung für dieses Fachgebiet die MRT keinerlei Erwähnung findet und auch dementsprechend keine Richtzahlen in den Weiterbildungsrichtlinien des Gebiets vorgeschrieben werden.

Zudem ist ergänzend darauf hinzuweisen, dass die Einführung einer Zusatz-Weiterbildung nicht dazu führt, dass eine Methode von dem Fachgebiet, auf das sich die Zusatz-Weiterbildung bezieht, automatisch umfasst ist. $§ 2$ Abs. 4 S. 4 der Weiterbildungsordnung der BLÄK bestimmt insoweit:

„Die Gebietsgrenzen werden durch eine Zusatz-Weiterbildung nicht erweitert."

Das bedeutet einerseits, dass die Einführung der Zusatz-Weiterbildung nicht dazu führt, dass die MRT als Methode vom Gebiet des Facharztes/Fachärztin für Orthopädie und Unfallchirurgie umfasst ist. Dass fakultativ eine Zusatzqualifikation zur Durchführung von MRT-Untersuchungen erworben werden kann, ändert dagegen nichts daran, dass die Weiterbildung zum Orthopäden diese Qualifikation nicht fordert (vgl. BSG, Urteil vom 02.04.2014, Az.: B 6 KA 24/13 R, Kardiologie).

Darüber hinaus gewährt eine Zusatz-Weiterbildung nur eine nach Organen differen- zierte fachliche Kompetenz (vgl. BVerfG, Beschluss vom 02.05.2018, Az.: 1 BvR 3042/14). Wie die Bezeichnung „ZusatzWeiterbildung Magnetresonanztomographie - fachgebunden - “ bereits deutlich macht, gewährt sie lediglich eine Berechtigung zur Durchführung der Diagnostik im Rahmen der Inhalte des Fachgebietes. Der Facharzt/Fachärztin für Orthopädie und Unfallchirurgie ist daher strikt auf die MRTDiagnostik zur „Vorbeugung, Erkennung, konservative und operative Behandlung, Nachsorge und Rehabilitation von chirurgischen Erkrankungen, Verletzungen und Verletzungsfolgen sowie angeborenen und erworbenen Formveränderungen und Fehlbildungen der Gefäße, der inneren Organe einschließlich des Herzens, der Stütz- und Bewegungsorgane sowie der Wiederherstellungs- und Transplantationschirurgie“ beschränkt.

Dies führt im Ergebnis dazu, dass eine Zusatz-Weiterbildung gegenüber einer Facharztausbildung nicht gleichwertig ist (vgl. BVerfG, Beschluss vom 02.05.2018, Az.: 1 BvR 3042/14). Dies gilt insbesondere im Verhältnis zum Fachgebiet der Radiologie, welches weiterhin die universelle Berechtigung zur Durchführung von MRTUntersuchungen für alle Organbereiche besitzt, da nach der MWBO sowie der Weiterbildungsordnung für die Ärzte Bayerns die Inhalte der Zusatz-Weiterbildung „ZusatzWeiterbildung Magnetresonanztomographie - fachgebunden - “ integraler Bestandteil der Weiterbildung zum Facharzt für Radiologie sind und zudem die Weiterbildungsinhalte, die Weiterbildungszeiten und die Richtzahlen in den Weiterbildungsrichtlinien im Fachgebiet Radiologie deutlich umfangreicher sind, als im Rahmen der Zusatz-Weiterbildung.

Das Bundessozialgericht hat zudem die zutreffende Auffassung vertreten, dass die durch die Zusatz-Weiterbildung erworbene Qualifikation derjenigen eines Radiologen bereits deshalb nicht vergleichbar ist, weil sie nach der MWBO bei einem weiterbildungsberechtigten Radiologen erworben werden muss (vgl. BSG, Urteil vom 02.04.2014, Az.: B 6 KA 24/13 R). Diese Vorgabe findet sich auch in der Zusatz-Weiterbildung „Magnetresonanztomographie - fachgebunden -“ der BLÄK. 


\section{Die Verpflichtung zur Beschränkung auf das Fachgebiet}

Soweit eine Fachfremdheit besteht, haben die Ärztekammern die gesetzliche Pflicht zur Fachgebietsbeschränkung in den Weiterbildungsordnungen - unter Berücksichtigung der Qualifikationen - auszugestalten. Die maßgebliche Regelung in $\S 2$ Abs. 2 der Weiterbildungsordnung der BLÄK lautet:

„(2) Gebiet ist ein definierter Teil in einer Fachrichtung der Medizin. Die Gebietsdefinition bestimmt die Grenzen für die Ausübung der fachärztlichen Tätigkeit. Die in der Facharztkompetenz vorgeschriebenen Weiterbildungsinhalte beschränken nicht die Ausübung der fachärztlichen Tätigkeit im Gebiet.“

Die gesetzliche Verpflichtung zur Aufnahme dieser Regelung in die Weiterbildungsordnung regelt in Bayern Art. 34 Abs. 1 Heilberufe-Kammergesetz (HKaG) in der Fassung vom 12.07.2018 (GVBI. S. 545):

„(1) Wer eine Gebietsbezeichnung führt, darf grundsätzlich nur in dem Gebiet, wer eine Teilgebietsbezeichnung führt, muss auch in dem Teilgebiet tätig sein, dessen Bezeichnung er führt."

Wie bereits in Teil 1 dieses Beitrages („Verfassungsrechtliche Grundlagen des ärztlichen Weiterbildungsrechts“) dargestellt, beruht die Verpflichtung zur Begrenzung der Arzttätigkeit auf ein bestimmtes Gebiet auf dem sog. Facharzt-Beschluss des BVerfG vom 09.05.1972 (BVerfGE 33, 125 ff.). Die Entscheidung wurde durch einen Nichtannahmebeschluss des BVerfG vom 17.06.1999 (Az.: 1 BvR 1500/97) bestätigt, in dem das Gericht zum Überweisungsvorbehalt bei Ärzten für Labormedizin Stellung genommen hat:

„Die Begrenzung der Facharzttätigkeit auf das eigene Fach kann durch vernünftige Erwägungen des Gemeinwohls eine Einschränkung der freien Berufsausübung grundsätzlich rechtfertigen (vgl. BVerfGE 33, $125<167>$ ). Soweit die Gerichte in den angegriffenen Entscheidungen gerade aus den Besonderheiten eines diagnostischen medizinischen Fachgebiets und der in der Weiterbildungsordnung verankerten Definition von Laboratoriumsmedizin als „Beratung und Unterstützung der in der Vorsorge und in der Krankenbehandlung tätigen Ärzte bei der Erkennung von Krankheiten und ihren Ursachen ... " einen Überweisungsvorbehalt abgeleitet haben, ist dies von Verfassungs wegen nicht zu beanstanden (vgl. auch den Beschluß nach \$93a BVerfGG vom 29. Mai 1978-1 BvR 77/75 -, SozR 2200 $\S 368 n$ RVO Nr. 16).“

Durch Beschluss vom 01.02.2011 (Az.: 1 BvR 2383/10 = MedR 2011, 572 ff.) hat des BVerfG bestätigt, dass inhaltliche Einwände gegen die Regelung, grundsätzlich nur in dem Gebiet der Gebietsbezeichnung tätig zu werden, unter Berücksichtigung der hierzu ergangenen Rechtsprechung des Gerichts nicht erkennbar sind. Ausnahmen hiervon hat das Bundesverfassungsgericht in seinem Beschluss vom 01.02.2011 nur bei geringfügig ausgeübten fachgebietsfremden Tätigkeiten zugelassen (Anteil unter $5 \%$ ). In den vorliegenden gerichtlichen Verfahren geht es jedoch um eine regelhafte Leistungserbringung im Bereich der MRT durch Fachärzte für Orthopädie und Unfallchirurgie und damit um eine in ihrer Art und ihrem Umfang erhebliche, dauerhafte fachfremde Tätigkeit, die ohne entsprechende Qualifikationsnachweise nicht zulässig ist.

Aus einer Verletzung der Beschränkung auf das Fachgebiet nach Art. 34 Abs. 1 HKaG folgt nach der Entscheidung des BVerfC vom 01.02.2011 zugleich die Befugnis und die Verpflichtung für Ärztekammern und Berufsgerichte, einen solchen schuldhaften Verstoß eines Arztes gegen seine Berufspflichten zu ahnden. Dies ergibt sich in Bayern aus Art. 66 Abs. $1 \mathrm{HKaG}$ und in anderen Bundesländern aus vergleichbaren Vorschriften in den Heilberufs- und Kammergesetzen (z. B. §59 Abs. 1 Heilberufsgesetz NRW).

\section{Keine Befreiung von der Gebietsbeschränkung aus Verhältnismäßigkeitsgründen}

Das BVerfG hat in der zitierten Entscheidung vom 01.02.2011 zudem betont, dass der Grad der Schwierigkeit der Leistungserbringung ein Indiz dafür ist, einen Arzt nicht von der Beschränkung auf das Fachgebiet zu befreien:
„Besondere individuelle Umstände, die es gebieten würden, den Beschwerdeführer generell von der Gebietsbeschränkung auszunehmen, sind nicht erkennbar. Soweit der Beschwerdeführer sich auf den hohen Schwierigkeitsgrad der Behandlungen auf seinem Facharztgebiet, die ihn zur Vornahme der fachfremden Operationen in besonderem Maße qualifizierten, beruft, spricht dies nicht für, sondern vielmehr gegen eine komplette Befreiung von der Gebietsbeschränkung. Denn wenn es zutrifft, dass die Eingriffe im Bereich seines Fachgebiets besonders schwierig sind, erfordern sie eine ständige Übung, die fehlen würde, wenn die fachgebietsfremde ärztliche Tätigkeit zahlenmäßig überhand nähme.“

Die Tatsache, dass die Anwendung der MRT, trotz der fehlenden ionisierenden Strahlung generell nicht ungefährlich ist und zu körperlichen Schäden bei den Patienten führen kann, ist durch die „Empfehlungen zur sicheren Anwendung magnetischer Resonanzverfahren in der medizinischen Diagnostik“ der Strahlenschutzkommission vom 19./20. September 2002, (BAnz. Nr. 72 vom 12. April 2003), der Stellungnahme der Bundesärztekammer in ihren Leitlinien zur Qualitätssicherung der Magnet-Resonanz-Tomographie vom 29.01.1999 (veröffentlicht im Deutschen Ärzteblatt 97, Heft 39, 29. September 2000, Seite A 2557-A 2568) und der Kernspintomographie-Vereinbarung nach $\S 135$ Abs. 2 SGB V belegt. Neben etwaigen körperlichen Beeinträchtigungen der Patienten durch eine medizinisch nicht indizierte Anwendung der MRT sind insbesondere Fehlbefundungen aufgrund mangelnder Qualifikation des anwendenden Arztes und die sich hieraus ergebenden fehlerhaften Indikationsstellungen für Folgetherapien zu nennen. Gerade das bei nichtradiologischen Fachgebieten nicht vorhandene Überweisungserfordernis führt in der GKV zu einem Ausschluss des „Mehraugenprinzips“, sodass die Anwendung der MRT - verglichen mit der Anwendung durch qualifizierte Radiologen - hier eine weitaus größere Gefahrenquelle in sich birgt.

Die Bundesärztekammer weist in ihren Leitlinien darauf hin, dass die MRT gegenüber allen anderen Schnittbildverfahren die Fähigkeit des Arztes erfordert, „die Messbedingungen und die Messparameter der Fragestellung“ anzupassen, um über die 
Auswertung der Schnittbilder und der anderen Messergebnisse erfolgreich zu einem aussagekräftigen Befund zu gelangen.

Die besonderen Gründe für eine Notwendigkeit einer qualitätsgesicherten Durchführung im Bereich der MRT hat das BVerfG für die Regelung in der GKV bestätigt, warum aufgrund der gesetzlichen Anordnung in § 135 Abs. 2 Satz 4 SGB V eine Konzentration dieser diagnostischen Leistungen auf das Fachgebiet der Radiologie erfolgt ist. Hierzu gehören die Regelungen über die Überweisungsabhängigkeit („Verbot der Selbstzuweisung“) aller Leistungen von Radiologen im Bundesmantelvertrag, die nicht nur zu einer Wirtschaftlichkeit der Leistungserbringung, sondern auch zu einer erhöhten Qualitätssicherung durch das „Mehraugenprinzip“ führen. Diese Gründe hat das BSG bereits in seiner Entscheidung vom 31.01.2001 (Az.: B 6 KA 24/00 R) überzeugend dargelegt. Sie machen deutlich, dass die besondere Qualifikation des Fachgebietes Radiologie in der kernspintomografischen Diagnostik deutlich über der aller anderen Fachgebiete liegt:

„Der Zuordnung spezieller diagnostischer Verfahren zu sog diagnostischen Methodenfächern oder jedenfalls zu entsprechend intensiv ausgebildeten Ärzten, wie dies in wesentlichen Teilen des Laborbereichs, der Radiologie und Nuklearmedizin sowie der Pathologie realisiert worden ist, liegen Gemeinwohlerwägungen von beachtlichem Gewicht zugrunde. Gerade im hier betroffenen Bereich der Kernspintomographie kommt der Qualitätssicherung erhebliche Bedeutung zu, da - worauf schon hingewiesen worden ist - die Fehlermöglichkeiten besonders groß sind (vgl. die oben zitierte Präambel der Leitlinien der BÄK zur Qualitätssicherung, DÄ 2000, C-1936). Die Konzentration der Kernspintomographien bei dafür speziell qualifizierten Ärzten bewirkt, daß diese viele derartige Untersuchungen durchführen und dadurch in deren Durchführung sowie Auswertung besonders erfahren und geübt sind. Das Erfordernis umfassender Ausbildung in diagnostischer Radiologie soll gewährleisten, daß der Arzt das gesamte Spektrum möglicher radiologischer Untersuchungen überblickt (Röntgen, Computer-, Kernspintomographie usw.) und beurteilen kann, ob möglicherweise eine andere Untersuchungsmethode als die Kernspintomographie im konkreten Fall geeigneter, schonender und/oder kostensparender ist. Er kann dementsprechend die an ihn überweisenden Ärzte bei der Auswahl der geeigneten Untersuchungsmethode fundiert beraten. Durch die intensive Ausbildung in kernspintomografischer Diagnostik wird erreicht, daß der Arzt auch pathologische Befunde, die nicht den Untersuchungsanlass bilden, im Bild erkennen, identifizieren und interpretieren kann (sog Zufallsbefunde). Ferner führt die Konzentration der diagnostischen Methodik bei bestimmten Ärzten zu einer Arbeitsteilung is des sog Mehraugenprinzips, dh daß die Diagnostik einem anderen Arzt obliegt als die anschließende Therapie. Eine solche Diagnostik, die unabhängig von einem eventuellen Interesse an der Therapie erfolgt, dient zum einen der optimalen $\mathrm{Pa}$ tientenversorgung, zum anderen dem sparsamen Einsatz der Leistungsressourcen. So wird der Möglichkeit vorgebeugt, daß der Behandler den Befund ausdehnend interpretiert und damit nicht unbedingt notwendige kostenträchtige Behandlungsmaßnahmen rechtfertigt. Diese Gesichtspunkte haben bei Untersuchungen, die - wie das bei Kernspintomographien der Fall ist - sehr komplex und zudem kostspielig sind, besonders große Bedeutung. Mithin dient die Konzentration kernspintomografischer Leistungen bei dafür speziell und umfassend qualifizierten Ärzten gewichtigen Gemeinwohlbelangen, nämlich - wie gesagt - sowohl der Gesundheit der Versicherten (zu diesem Gemeinschaftsgut s. zuletzt BSG SozR 3-2500 §135 Nr 15 S 78) als auch der finanziellen Stabilität und Funktionsfähigkeit der gesetzlichen Krankenversicherung (vgl dazu zB BSGE 82, 41, 45 = SozR 3-2500 § 103 Nr2 S 15 mwN). “

Im Ergebnis ist daher festzustellen, dass bei der Leistungserbringung der MRT, wie bei allen diagnostischen Verfahren, die Befundung der technischen Leistung als ärztlicher Leistungsanteil im Vordergrund steht. Die technische Durchführung der Untersuchung kann hingegen auf qualifiziertes medizinisch-technisches Fachpersonal (MTRA, MFA) delegiert werden. Die korrekte Befundung der Aufnahmen ist jedoch nur gewährleistet, wenn der Arzt über eine qualifizierte Weiterbildung in der Durchführung dieses Verfahrens verfügt. Dies ist bei Fachärzten für Orthopädie und Unfallchirurgie, wie auch bei allen anderen Fachärzten außerhalb der Radiologie, nur dann und beschränkt auf das jeweilige Gebiet gegeben, wenn sie die vorgeschriebene ZusatzWeiterbildung MRT - fachgebunden durchlaufen haben, denn hier wird eine 24-monatige Weiterbildung bei einem Weiterbilder für Radiologie vorgeschrieben, in der insbesondere die Durchführung und Befundung gebietsbezogener Untersuchungen mittels Magnetresonanztomographie mit einer Richtzahl von 1.000 selbständig durchgeführter und befundeter Untersuchungen vorgeschrieben wird. Diese Richtzahl ist bei Facharztgruppen, wie Orthopäden und Kardiologen, die im Rahmen ihrer Facharztausbildung i. d. R. keinerlei praktische Kenntnisse und Erfahrungen im Bereich der Schnittbilddiagnostik erworben haben, erforderlich, damit sie sich mit diesem diagnostischen Verfahren prinzipiell vertraut machen können. Eine umfassende Ausbildung in der Methode der MRT für alle Körperregionen, wie im Fachgebiet der Radiologie, ist damit gleichwohl nicht verbunden.

Für das Fachgebiet Radiologie ist für alle Körperregionen demgegenüber eine Richtzahl von 3.000 Untersuchungen vorgeschrieben. Diese Vorgaben der Weiterbildungsordnungen und Weiterbildungsrichtlinien belegen, dass es sich bei der Durchführung der MRT keineswegs um ein einfaches diagnostisches Verfahren handelt. Andernfalls hätte man für die MRT auf die Vorgabe von Richtzahlen verzichten können, wie dies in den Weiterbildungsrichtlinien mit der Angabe „BK“ (Basiskompetenz) für einfache Verfahren geschieht. Die Angabe „BK“ in der Spalte „Richtzahl“ bedeutet, dass der Erwerb von Kenntnissen, Fertigkeiten und Erfahrungen gefordert ist, ohne dass hierfür eine festgelegte Mindestzahl nachgewiesen werden muss.

Es ist daher aus Gründen der Patientensicherheit erforderlich, dass die Fachgebietsgrenzen in Bezug auf die Methode der Magnetresonanztomographie eingehalten werden. Nur eine enge Verflechtung von eingehenden Kenntnissen, Erfahrungen und Fertigkeiten in der MRT ermöglichen eine fach- und fallgerechte Indikationsstellung, optimale Durchführung und eine korrekte differentialdiagnostische Befundung im Kontext anderer bildgebender Verfahren.

Die MRT ist ein technisch aufwendiges, nicht risikofreies und kostenintensives Verfahren, zu dessen Durchführung die nötigen Erfahrungen und Fertigkeiten nur im Rahmen einer geordneten Weiterbildung der Ärzte- 
kammern erlernt werden können. Gerade die Abwägung, wann eine MRT-Untersuchung im Vergleich zu anderen bildgebenden Verfahren indiziert bzw. kontraindiziert ist und welche Untersuchungstechnik in Abhängigkeit zur Indikation zu wählen ist, erfordert regelmäßig die vorgesehene Weiterbildungszeit von 24 Monaten in "ganztägiger hauptberuflicher Stellung“ bei einem Weiterbilder für Radiologie und die Durchführung und Befundung von mindestens 1.000 Untersuchungen im Rahmen der Zusatz-Weiterbildung MRT - fachgebunden -, wenn Fachärzte für Orthopädie und Unfallchirurgie diese Untersuchungsleistungen erbringen wollen.

\section{Abrechnung von MRT- Leistungen durch Fachärzte/ Fachärztinnen für Orthopädie und Unfallchirurgie nach der GOÄ}

Nach § 4 Abs. 2 Gebührenordnung für Ärzte (GOÄ) kann der Arzt nur für selbstständige ärztliche Leistungen Gebühren abrechnen, die er selbst erbracht hat oder die unter seiner Aufsicht nach fachlicher Weisung erbracht wurden. Die notwendige "fachliche Weisung" setzt aber voraus, dass der abrechnende Arzt selbst über die notwendige fachliche Qualifikation zur Erbringung der delegierten bzw. veranlassten Leistungen verfügt. Kann der Arzt die Verrichtung mangels entsprechender weiterbildungsrechtlicher Qualifikation nicht fachgerecht selbst erbringen, ist er zur Abrechnung nicht berechtigt. Voraussetzung für einen Vergütungsanspruch nach § 4 Abs. 2 GOÄ ist daher, dass die betreffende Leistung auf der Grundlage der aktuellen Weiterbildungsordnung als fachgebietskonform angesehen werden kann (vgl. Klakow-Franck (Hrsg), Kommentar zur Gebührenordnung für Ärzte (GOÄ), 2019, § 4 Rn. 9; Uleer, Miebach, Patt, Abrechnung von Arzt- und Krankenhausleistungen, 2000, §4 GOÄ, S. 36, 39; Vorstand der Bundesärztekammer, DÄBI. 1996, A-2720; Cramer und Henkel, MedR 2004, S. 593, 596 m.w.N.).

Der Kommentar zur GOÄ von KlakowFranck führt hierzu folgendes aus:

"Soweit die Weiterbildungsordnung einer Landesärztekammer zur Erbringung bestimmter
Leistungen spezielle Kenntnisse, Fertigkeiten und Fähigkeiten vorschreibt, sind diese auch Voraussetzung für die Liquidation dieser Leistungen nach der GOÄ.“

(vgl. Klakow-Franck (Hrsg), Kommentar zur Gebührenordnung für Ärzte (GOÄ), 2019, $\S 4$ Rn. 9)

Begründet wird dies damit, dass der Arzt durch das Führen einer Facharztbezeichnung nach der Weiterbildungsordnung bei seinen Patienten das Vertrauen einer entsprechenden fachlichen Qualifikation erweckt. Insofern wird auf die Rechtsprechung zum „Facharztstandard“ im Haftungsrecht ausdrücklich hingewiesen (BGH, Beschl. v. 09.06.2009, Az.: VI ZR 261/08). Indirekt würde diese Auffassung auch durch den Beschluss des BVerfG vom 01.02.2011 bestätigt, wonach nur eine in geringfügigem Umfang ausgeübte fachgebietsfremde Tätigkeit von weniger als 5 Prozent berufsrechtlich nicht zu sanktionieren sei (BVerfG, Beschl. v. 01.02.2011, Az.: 1 BvR 2383/10). Die entgegenstehende Auffassung von Taupitz wird demgegenüber ausdrücklich abgelehnt (vgl. Taupitz MedR 1996, 498, 500).

Fachärzte/Fachärztinnen für Orthopädie und Unfallchirurgie, die den Nachweis der Erlangung der fachlichen Kompetenz in fachgebundener MRT durch den Abschluss der Zusatz-Weiterbildung MRT - fachgebunden - nicht erbracht haben, sind folglich zur Abrechnung dieser Leistungen nach der GOÄ nicht berechtigt. Diese Rechtsauffassung hat das OLG Celle in Bezug auf die Abrechnung von MRT-Untersuchungen durch Fachärzte für Orthopädie in einer Entscheidung vom 22.10.2007 (Az.: $1 \cup$ 77/07) vertreten, in dem das Gericht einem privaten Krankenversicherungsunternehmen gegen einen Facharzt für Orthopädie aus übergegangenem Recht gemäß $\S \S 812$ Abs. 1, 398 BGB, 67 VVG einen Anspruch auf Rückforderung des von ihrem Versicherungsnehmer gezahlten Honorars für MRT-Leistungen zugebilligt hatte:

„a) Die Bezahlung der streitgegenständlichen Behandlungskosten für die Durchführung der MRT-Untersuchungen durch den Beklagten als Facharzt für Orthopädie erfolgte seitens der Versicherungsnehmer der Klägerin ohne rechtlichen Grund. Denn ein Anspruch aus $\S \S 611,612$ BGB i.V. mit den Regelungen der
GOÄ auf Zahlung des ärztlichen Honorars für die Durchführung dieser Maßnahmen stand dem Beklagten gegen die Versicherungsnehmer der Klägerin nicht zu. Gem. §1 Abs. 2 Satz 1 GOÄ darf der Arzt Vergütungen nur für Leistungen berechnen, die nach den Regeln der ärztlichen Kunst für eine medizinisch notwendige ärztliche Versorgung erforderlich sind. §1 Abs. 2 Satz 1 GOÄ greift ein berufsrechtliches Leitbild für die ärztliche Tätigkeit auf und verknüpft damit den Vergütungsanspruch des Arztes. Auch im Rahmen einer Privatbehandlung ist dieser grundsätzlich, von begründeten Ausnahmefällen wie etwa Notfallbehandlungen abgesehen, an die Grenzen seines medizinischen Fachgebietes gebunden. Nur dann können seine Leistungen den Regeln der ärztlichen Kunst entsprechen. Erbringt der Arzt also fachfremde Leistungen, ohne dass dies ausnahmsweise - etwa in Notfällen - gerechtfertigt ist, hat er keinen Honoraranspruch gegen den Patienten (Uleer/Miebach/ Patt, Abrechnung von Arzt- und Krankenhausleistungen, Kommentar, 3. Aufl., § $1 \mathrm{GOÄ}$ Rnrn. 10,13 m.w.N.). So aber liegt es hier. Entgegen der Auffassung des Landgerichts ist davon auszugehen, dass der Beklagte mit der Vornahme der MRT-Untersuchungen an den Versicherungsnehmern der Klägerin damals im Jahre 2004 für ihn als zugelassenen Orthopäden fachfremde Leistungen erbracht hat."

Im Hinblick auf die zwischenzeitlich eingeführten Vorschriften über den Behandlungsvertrag nach §§ 630a ff. BGB ist anzumerken, dass sich der Vergütungsanspruch des Arztes aktuell nach den $\S \S 630$ a Abs. 1, 630b, 612 BGB und nicht mehr nach den dienstvertraglichen Regelungen in $\S \S 611,612$ BGB richtet. Nach diesem Urteil des OLG Celle besteht daher kein Liquidationsanspruch von Orthopäden für MRT-Leistungen. Die Landgerichte Regensburg und Landshut haben diese Entscheidung im Rahmen ihrer Urteilsfindung nicht berücksichtigt.

\section{Ergebnis}

Im Ergebnis ist daher die den Landgerichten Regensburg und Landshut mitgeteilte Rechtsauffassung des Geschäftsführers der BLÄK unzutreffend, wonach nach der gegenwärtigen und der früheren Weiterbildungsordnung „der Orthopäde im Rahmen seines Gebietes auch Magnetresonanztomographie berufsrechtlich gebietskonform durchführen darf." 
Vielmehr ist die Magnetresonanztomographie weiterhin nicht Teil der Weiterbildung dieses Fachgebietes. Zwar kann der Facharzt/Fachärztin für Orthopädie und Unfallchirurgie diese Methode im Rahmen der „Zusatz-Weiterbildung Magnetresonanztomographie - fachgebunden -“ gebietsbezogen erlernen. Da jedoch nach §2 Abs. 4 der Weiterbildungsordnung der BLÄK die Gebietsgrenzen fachärztlicher Tätigkeiten durch die Zusatz-Weiterbildungen nicht erweitert werden, stellt die MRT für das Fachgebiet der Orthopädie lediglich eine zusätzlich erlangbare fachliche Kompetenz dar, die jedoch nicht bereits automatisch mit Erwerb der Facharztbezeichnung im Gebiet erworben wird.

Dies führt dazu, dass die Erbringung und Abrechnung von MRT-Leistungen durch Fachärzte/Fachärztinnen für Orthopädie und Unfallchirurgie sowie für die früheren Fachärzte für Orthopädie als fachgebietsfremd anzusehen ist. In jedem Fall fehlt ihnen die nach der WBO 2018 erforderliche kognitive Methodenkompetenz und die Handlungskompetenz zur Durchführung von MRT-Untersuchungen. Die Frage der Berechtigung zur Erbringung dieser Leistungen im Einzelfall kann sich daher nur nach Ableistung der Zusatz-Weiterbildung Magnetresonanztomographie - fachgebunden - ergeben und setzt voraus, dass der Facharzt/Fachärztin für Orthopädie und Unfallchirurgie die fachliche Kompetenz in fachgebundener MRT nach Ableistung der vorgeschriebenen Weiterbildungszeit und Weiterbildungsinhalte erworben hat (so auch OLG Celle, Urteil vom 22.10.2007, Az.: 1 U 77/07).

Soweit der Erwerb der Zusatz-Weiterbildung Magnetresonanztomographie - fachgebunden - durch einen Facharzt/Fachärztin für Orthopädie und Unfallchirurgie nicht nachgewiesen worden ist, stellt die Erbringung dieser Leistungen einen Verstoß gegen § 2 Abs. 2 S. 2 MWBO 2018 sowie der entsprechenden Vorschriften der Weiterbildungsordnungen der Landesärztekammern (vgl. z. B. §2 Abs. 2 S. 2 Weiterbildungsordnung für die Ärzte Bayerns) und der Bestimmungen in den Heilberufs- und Kammergesetzen der Länder (z. B. §34 Abs. 2 des bayerischen Heilberufe-Kammergesetz HKaG) dar, wonach derjenige, der eine Gebietsbezeichnung führt, grundsätzlich nur in dem Gebiet tätig sein darf, dessen Bezeichnung er führt.

Fachärzte/Fachärztinnen für Orthopädie und Unfallchirurgie, die MRT-Leistungen erbringen, ohne dass sie über eine ZusatzWeiterbildung Magnetresonanztomographie - fachgebunden - verfügen, erlangen daher auch keinen Vergütungsanspruch gegenüber den Patienten nach $§ 4$ Abs. 2 GOÄ.

Prof. Dr. Peter Wigge

Rechtsanwalt

Fachanwalt für Medizinrecht

Rechtsanwälte Wigge

Scharnhorststraße 40

48151 Münster

Telefon: (0251) 53 595-0

Telefax: (0251) 53 595-99

E-Mail: kanzlei@ra-wigge.de

www.ra-wigge.de 